\title{
26355 - A RETROSPECTIVE STUDY OF EPIDURALS POST-LUNG TRANSPLANTATION
}

\section{Danny Chen MD, Ban Tsui, MD; University Of Alberta, Edmonton, ALBERTA, Canada}

INTRODUCTION: Thoracic epidural analgesia (TEA) is a modality used to achieve good analgesia and improved respiratory function after major surgeries, including lung transplants with the associated thoracotomy. Subsequently, quicker extubation and shorter ICU stays can help patients avoid complications. The Göteborg Lung Transplant Group in Sweden reported that early extubation was related to routine preoperative TEA and provision of continuous positive airway pressure (CPAP) following endotracheal tube removal.1 Despite this, there is the risk of perioperative epidural complications, including rare but devastating spinal cord damage. In addition, the cases that need cardiopulmonary bypass require anticoagulation which can potentially cause hematoma formation. 2 With these concerns, the patients at our institution do not receive TEA until 1-2 days postoperatively. To access the merit of our protocol, we would like to study TEA efficacy, in relation to standard patient controlled analgesia (PCA), regarding: placement safety, earlier extubation, pain management, and length of hospital stay. METHODS: After ethics approval, the first consecutive 31 charts of lung transplant patients starting from January 2002 were retrospectively examined. Two patients passed away due to surgical and/or medical causes prior to receiving PCA or TEA and were excluded. The parameters investigated include: patient demographics and history, surgical details, TEA and PCA information, coagulation parameters, perioperative complications, extubation timing, and discharge dates.

RESULTS: Table 1 summarizes the chart data collected from 29 lung transplant patients and compares PCA with TEA. There was no significant difference in time to extubation between analgesic modalities. No significant differences were found in other study parameters. Most lung transplant patients required cardiopulmonary bypass.

DISCUSSION: Despite the suggestion of earlier extubation success for those patients who received TEA, there is no evidence of such benefits observed from this study. It is likely that the variables contributing to safe and shortened extubation and hospital stay are multi-factorial. When considering complications, anticoagulation is an important factor as a large proportion of lung transplant patients required cardiopulmonary bypass pumps. Using TEA postoperatively may be a way to avoid delaying cases due to bloody taps. Overall, the results of this study suggest POD ; 1 would be a reasonable time for TEA initiation. Furthermore, even though there were no major complications secondary to neuraxial techniques in this pilot study, there is theoretical risk involved in postoperative epidural placement into non-verbal, sedated and intubated patients. 2 Future work is planned to review all patients during the past five years to confirm such observations.

REFERENCES:

1. J of Cardiothoracic and Vasc Anes 1999; 13: 249-252

2. Anes Clinics of N America 2000; 18: 461-485 
Table 1: Patient Controlled Analgesia (PCA) versus Thoracic Epidural Analgesia (TEA) in 29 Lung Transplantation Patients

\begin{tabular}{|l|l|l|}
\hline & \multicolumn{1}{|l|}{ PCA Mean (range) } & TEA Mean (range) \\
\hline \# patients (n=29) & 7 & 22 \\
\hline Age (years) & $59.0(53-66)$ & $46.7(8-67)$ \\
\hline BMI (kg/m $\left.{ }^{2}\right)$ & $26.7(18-34)$ & $23.0(17-31)$ \\
\hline \% Single lung & 71.4 & 13.6 \\
\hline \% Double lung & 28.6 & 86.4 \\
\hline \% Bypass Pump & 42.9 & 72.7 \\
\hline INR Day 0 & $1.39(0.9-1.8)$ & $1.30(1.0-1.6)$ \\
Day 1 & $1.23(1.0-1.6)$ & $1.23(1.0-1.7)$ \\
Day 2 & $1.20(1.0-1.5)$ & $1.17(0.9-1.6)$ \\
Day 3 & $1.24(1.1-1.5)$ & $1.14(1.0-1.7)$ \\
\hline PTT Day 0 & $43.0(25-86)$ & $44.5(26-63)$ \\
Day 1 & $33.6(24-47)$ & $39.6(28-60)$ \\
Day 2 & $30.7(22-39)$ & $36.9(24-69)$ \\
Day 3 & $32.0(22-41)$ & $32.8(21-56)$ \\
\hline Initiation (Post-op day) & $1.8(0-8)$ & $0.7(0-2)$ \\
\hline Extubation (Post-op & $3.0(0-8)$ & $3.3(0-21)$ \\
day) & & \\
\hline Hospital stay (day): & & \\
- Single lung & $67.3(14-153)$ & $20.0(18-21)$ \\
- Double lung & $28.0(22-34)$ & $40.2(15-163)$ \\
\hline
\end{tabular}

